\title{
Temperature dependence of the density and excitations of dipolar droplets
}

\author{
S. F. Öztürk $\oplus^{*}$ \\ Department of Physics, Harvard University, Cambridge, Massachusetts 02138, USA \\ Enes Aybar $^{\dagger}$ and M. Ö. Oktel 10 \\ Department of Physics, Bilkent University, Ankara 06800, Turkey
}

(Received 1 July 2020; accepted 28 August 2020; published 21 September 2020)

\begin{abstract}
Droplet states of ultracold gases which are stabilized by fluctuations have recently been observed in dipolar and two-component Bose gases. These systems present an alternate form of equilibrium where an instability at the mean-field level is arrested by higher-order correlations, making the droplet states sensitive probes of fluctuations. In a recent paper, we argued that thermal fluctuations can play an important role for droplets even at low temperatures where the noncondensed density is much smaller than the condensate density. We used the Hartree-Fock-Bogoliubov theory together with the local density approximation for fluctuations to obtain a generalized Gross-Pitaevskii (GP) equation and solved it with a Gaussian variational ansatz to show that the transition between the low density and droplet states can be significantly modified by the temperature. In this paper, we first solve the same GP equation numerically with a time-splitting spectral method to check the validity of the Gaussian variational ansatz. Our numerical results are in good agreement with the Gaussian ansatz for a large parameter regime and show that the density of the gas is most strongly modified by temperature near the abrupt transition between a pancake-shaped cloud and the droplet. For cigar-shaped condensates, as in the recent Er experiments, the dependence of the density on temperature remains quite small throughout the smooth transition. We then consider the effect of temperature on the collective oscillation frequencies of the droplet using both a time-dependent Gaussian variational ansatz and real-time numerical evolution. We find that the oscillation frequencies depend significantly on the temperature close to the transition for the experimentally relevant temperature regime $(\simeq 100 \mathrm{nK})$.
\end{abstract}

DOI: 10.1103/PhysRevA.102.033329

\section{INTRODUCTION}

Ultracold bosonic gases form almost ideal, dilute, weakly interacting quantum systems. At temperatures much lower than the condensation temperature nearly all atoms share the collective ground-state wave function, and the effect of interactions can be accounted for by their self-consistent action on that wave function. This mean-field idea, captured by the Gross-Pitaevskii equation [1,2], qualitatively describes all the equilibrium properties of almost all trapped Bose gas experiments. The corrections to the dynamics described by the GP equation arise from particles which are above the condensate. The effect of this condensate depletion is detectable but remains small at low temperatures as long as the system remains dilute and weakly interacting.

An alternative scenario for equilibrium, in which the effect of fluctuations is crucial, can arise if the mean-field interactions describe an unstable system. The instability in the mean-field description can be cured by the inclusion of fluctuations, which remain small compared with mean-field

*sukrufurkanozturk@g.harvard.edu

${ }^{\dagger}$ Present address: ICFO-Institut de Ciencies Fotoniques, The Barcelona Institute of Science and Technology, 08860 Castelldefels (Barcelona), Spain. effects even when they provide stability. This scenario of fluctuation stabilized equilibrium was first suggested theoretically by Petrov [3] for two-component Bose mixtures and was recently observed experimentally [4-6]. For dipolar gases, droplet formation in the mean-field unstable regime was first reported experimentally, where follow-up experiments have definitively established the fluctuation based stabilization as their formation mechanism [7-10].

The theoretical description of the dipolar droplets have so far [11-15] relied on the generalized Gross-Pitaevskii equation for the condensate wave function

$$
\begin{aligned}
& {\left[-\frac{\hbar^{2} \nabla^{2}}{2 m}+U_{t r}(\mathbf{x})+\int d^{3} \mathbf{x}^{\prime} V_{\mathrm{int}}\left(\mathbf{x}, \mathbf{x}^{\prime}\right)\left|\Psi\left(\mathbf{x}^{\prime}\right)\right|^{2}\right] \Psi(\mathbf{x})} \\
& \quad=\left[\mu-\Delta \mu_{Q F}(\mathbf{x})\right] \Psi(\mathbf{x}),
\end{aligned}
$$

where the usual GP equation is modified by the quantum fluctuation contribution to chemical potential $\Delta \mu_{Q F}(\mathbf{x})$. This contribution is then assumed to be determined entirely in terms of the local condensate wave function as it would be found in a uniform gas

$$
\Delta \mu_{Q F}(\Psi(\mathbf{x})) \propto|\Psi(\mathbf{x})|^{3} .
$$

The exact coefficient linking $|\Psi|^{3}$ to $\Delta \mu_{Q F}$ depends on the infrared cutoff choice used the in the local density approximation (LDA), but all different choices 
used in the literature for this cutoff give results which are in agreement with observations, within experimental accuracy.

In a recent paper [16], we showed that the modified GP equation can be derived by using the Hartree-FockBogoliubov theory (HFBT) [17] together with the local density approximation for fluctuations. Such a treatment makes the assumptions underlying the generalized GP equation more transparent. We found that the above form of the generalized GP equation is valid only when the Hartree potential created by the depleted particles is negligible. This condition is satisfied in the dipolar droplet experiments, where the estimates for the number of depleted particles remain small even in the droplet regime.

The novel physical regime probed in the droplet experiments is evident in this set of approximations because the number of depleted particles is negligible compared with the number of condensed particles, but their interaction with the condensate wave function supplies the energy cost required for stability.

The derivation of the generalized GP equation from HFBT can be carried out at finite temperature $[16,18]$ without further complications. Quantum fluctuations are supplemented by thermal fluctuations, and in general the dynamics of such a system should be given in terms of a two-fluid model with separate equations for the condensate and normal components. However, at low enough temperatures, the total number of depleted atoms would still be much smaller than the number of atoms in the condensate, and the dynamics of the fluctuations can be described only in terms of the local condensate wave function. Thus, a finite-temperature version of the generalized GP equation was derived in which local chemical-potential correction is now a function of both the condensate density and the temperature $\Delta \mu_{Q F}(\Psi(\mathbf{x}), T)$. We solved this equation with a Gaussian variational ansatz and found that, even if the temperature depletion remains small, the condensate density may be strongly modified. The change in the local compressibility is particularly pronounced near the first-order transition to the droplet state.

In this paper, we first verify the conclusions obtained in Ref. [16], with a numerical solution of the generalized GP equation. We investigate the effects of temperatures which are much smaller than the BEC transition temperature, so that the thermal depletion density is much smaller than the condensate density but comparable to the quantum depletion. This temperature regime is likely to be relevant for both the Dy experiments by the Stuttgart group [7-9], and the Er experiments by the Innsbruck group [10]. Our numerical results show that the effect of temperature on the density is most prominent for sudden transitions to the droplet states, as obtained for initially oblate (pancake) clouds. For cigar-shaped (prolate) clouds, the transition to the droplet state is smooth, and the effect of temperature remains small throughout the transition. Based on this observation, we investigate whether collective oscillation frequencies show any dependence on temperature at these low temperatures. We use both an effective Lagrangian based on a Gaussian ansatz, and real-time numerical evolution to investigate the lowest-lying collective excitations of the droplet state. We find that the collective oscillation frequencies show strong dependence on temperature close to the first-order transition.

In the next section, we briefly summarize our HFBT method and give the resulting temperature-dependent generalized GP equation. Section III explains our numerical algorithm and gives the calculated equilibrium density profiles for the Er and Dy experiments. Section IV contains the temperature dependence of the lowest collective oscillation frequencies of the condensate obtained by both a variational Lagrangian method and numerical real-time evolution. A summary of the results and their relevance for the experiments on dipolar droplets are given in Sec. V.

\section{HARTREE FOCK BOGOLIUBOV THEORY AND THE MODIFIED GROSS PITAEVSKII EQUATION}

We consider bosonic atoms of mass $M$ in a confining potential $U_{\text {tr }}(\mathbf{x})$ with chemical potential $\mu$ and described by the Hamiltonian

$$
\begin{aligned}
\hat{H}= & \int d^{3} \mathbf{x} \hat{\psi}^{\dagger}(\mathbf{x})\left(-\frac{\hbar^{2} \nabla^{2}}{2 M}+U_{\mathrm{tr}}(\mathbf{x})-\mu\right) \hat{\psi}(\mathbf{x}) \\
& +\frac{1}{2} \iint d^{3} \mathbf{x} d^{3} \mathbf{x}^{\prime} \hat{\psi}^{\dagger}(\mathbf{x}) \hat{\psi}^{\dagger}\left(\mathbf{x}^{\prime}\right) V_{\mathrm{int}}\left(\mathbf{x}-\mathbf{x}^{\prime}\right) \hat{\psi}\left(\mathbf{x}^{\prime}\right) \hat{\psi}(\mathbf{x}),
\end{aligned}
$$

with the bosonic commutation $\left[\hat{\psi}(\mathbf{x}), \hat{\psi}^{\dagger}\left(\mathbf{x}^{\prime}\right)\right]=\delta\left(\mathbf{x}-\mathbf{x}^{\prime}\right)$ of field operators. The interaction potential between the particles have both a short-range and a long-range (dipolar) part:

$$
V_{\text {int }}(\mathbf{x})=g\left[\delta(\mathbf{x})+\frac{3 \epsilon_{d d}}{4 \pi|\mathbf{x}|^{3}}\left(1-3 \frac{z^{2}}{|\mathbf{x}|^{2}}\right)\right],
$$

where the short-range repulsion is expressed in terms of the $s$-wave scattering length $a_{s}$ as $g=4 \pi \hbar^{2} a_{s} / M$ and the relative strength of the dipolar interaction defines the dimensionless parameter $\epsilon_{d d}$.

We consider systems where most of the atoms occupy the condensate state. The total particle number $N$ is set by the chemical potential and $N_{0}$, the largest eigenvalue of the one-particle density matrix $\left\langle\hat{\psi}^{\dagger} \hat{\psi}\right\rangle$ is close to this number $N-N_{0} \ll N$. The part of the field operator corresponding to the condensate can then be safely approximated by the classical field $\Psi(\mathbf{x})$, the condensate wave function. The remaining part, which is referred to as the fluctuation operator, $\hat{\phi}(\mathbf{x})=\hat{\psi}(\mathbf{x})-\Psi(\mathbf{x})$ can be used to define the one-particle noncondensate density matrices. The direct noncondensate density is

$$
\tilde{n}\left(\mathbf{x}^{\prime}, \mathbf{x}\right)=\left\langle\hat{\phi}^{\dagger}\left(\mathbf{x}^{\prime}\right) \hat{\phi}(\mathbf{x})\right\rangle,
$$

and the anomalous noncondensate density is

$$
\tilde{m}\left(\mathbf{x}^{\prime}, \mathbf{x}\right)=\left\langle\hat{\phi}\left(\mathbf{x}^{\prime}\right) \hat{\phi}(\mathbf{x})\right\rangle .
$$

The aim of HFBT is to reduce the Hamiltonian to a system of self-consistent equations which describe the equilibrium values for the condensate wave function $\Psi(\mathbf{x})$ and the noncondensate densities $\tilde{n}, \tilde{m}$ [17]. This is achieved by assuming that the higher-order correlation functions can be factorized in 
terms of these three quantities, e.g.,

$$
\begin{aligned}
\hat{\phi}^{\dagger}(\mathbf{x}) \hat{\phi}^{\dagger}\left(\mathbf{x}^{\prime}\right) \hat{\phi}\left(\mathbf{x}^{\prime}\right) \approx & \tilde{m}^{*}\left(\mathbf{x}^{\prime}, \mathbf{x}\right) \hat{\phi}\left(\mathbf{x}^{\prime}\right)+\tilde{n}^{*}\left(\mathbf{x}^{\prime}, \mathbf{x}\right) \hat{\phi}^{\dagger}\left(\mathbf{x}^{\prime}\right) \\
& +\tilde{n}\left(\mathbf{x}^{\prime}\right) \hat{\phi}^{\dagger}(\mathbf{x}) .
\end{aligned}
$$

This procedure yields the GP equation

$$
\begin{aligned}
& \mathcal{L} \Psi(\mathbf{x})+\int d^{3} \mathbf{x}^{\prime} V_{\text {int }}\left(\mathbf{x}-\mathbf{x}^{\prime}\right) \tilde{n}\left(\mathbf{x}^{\prime}, \mathbf{x}\right) \Psi\left(\mathbf{x}^{\prime}\right) \\
& +\int d^{3} \mathbf{x}^{\prime} V_{\text {int }}\left(\mathbf{x}-\mathbf{x}^{\prime}\right) \tilde{m}\left(\mathbf{x}^{\prime}, \mathbf{x}\right) \Psi^{*}\left(\mathbf{x}^{\prime}\right)=0,
\end{aligned}
$$

where $\quad \mathcal{L}=\left[-\hbar^{2} \nabla^{2} / 2 M-\mu+U_{\text {tr }}(\mathbf{x})+\int d^{3} \mathbf{x}^{\prime} V_{\text {int }}(\mathbf{x}-\right.$ $\left.\left.\mathbf{x}^{\prime}\right)\left|\Psi\left(\mathbf{x}^{\prime}\right)\right|^{2}+\int d^{3} \mathbf{x}^{\prime} V_{\text {int }}\left(\mathbf{x}-\mathbf{x}^{\prime}\right) \tilde{n}\left(\mathbf{x}^{\prime}\right)\right], \quad$ displaying the dependence of the condensate wave function on the noncondensate densities.

To complete the self-consistent loop, noncondensate densities must be calculated in terms of the condensate wave function. This is achieved by obtaining the Bogoliubov-de Gennes $(\mathrm{BdG})$ equations for excitations,

$$
\begin{aligned}
E_{j} u_{j}(\mathbf{x})= & \mathcal{L}_{0} u_{j}(\mathbf{x})+\int d^{3} \mathbf{x}^{\prime} V_{\mathrm{int}}\left(\mathbf{x}-\mathbf{x}^{\prime}\right) \Psi^{*}\left(\mathbf{x}^{\prime}\right) \Psi(\mathbf{x}) u_{j}\left(\mathbf{x}^{\prime}\right) \\
& -\int d^{3} \mathbf{x}^{\prime} V_{\mathrm{int}}\left(\mathbf{x}-\mathbf{x}^{\prime}\right) \Psi\left(\mathbf{x}^{\prime}\right) \Psi(\mathbf{x}) v_{j}\left(\mathbf{x}^{\prime}\right), \\
-E_{j} v_{j}(\mathbf{x})= & \mathcal{L}_{0} v_{j}(\mathbf{x})+\int d^{3} \mathbf{x}^{\prime} V_{\mathrm{int}}\left(\mathbf{x}-\mathbf{x}^{\prime}\right) \Psi\left(\mathbf{x}^{\prime}\right) \Psi^{*}(\mathbf{x}) v_{j}\left(\mathbf{x}^{\prime}\right) \\
& -\int d^{3} \mathbf{x}^{\prime} V_{\text {int }}\left(\mathbf{x}-\mathbf{x}^{\prime}\right) \Psi^{*}\left(\mathbf{x}^{\prime}\right) \Psi^{*}(\mathbf{x}) u_{j}\left(\mathbf{x}^{\prime}\right),
\end{aligned}
$$

where $\quad \mathcal{L}_{0}=\left[-\hbar^{2} \nabla^{2} / 2 M-\mu+U_{\text {tr }}(\mathbf{x})+\int d^{3} \mathbf{x}^{\prime} V_{\text {int }}(\mathbf{x}-\right.$ $\left.\left.\mathbf{x}^{\prime}\right)\left|\Psi\left(\mathbf{x}^{\prime}\right)\right|^{2}\right]$. The solutions for the amplitudes $u, v$ can be summed to give the noncondensate densities

$$
\begin{aligned}
\tilde{n}\left(\mathbf{x}^{\prime}, \mathbf{x}\right)= & \sum_{j}\left\{v_{j}\left(\mathbf{x}^{\prime}\right) v_{j}^{*}(\mathbf{x})\right. \\
& \left.+N_{B}\left(E_{j}\right)\left[u_{j}^{*}\left(\mathbf{x}^{\prime}\right) u_{j}(\mathbf{x})+v_{j}\left(\mathbf{x}^{\prime}\right) v_{j}^{*}(\mathbf{x})\right]\right\}, \\
\tilde{m}\left(\mathbf{x}^{\prime}, \mathbf{x}\right)= & -\sum_{j}\left\{u_{j}\left(\mathbf{x}^{\prime}\right) v_{j}^{*}(\mathbf{x})\right. \\
& \left.+N_{B}\left(E_{j}\right)\left[v_{j}^{*}\left(\mathbf{x}^{\prime}\right) u_{j}(\mathbf{x})+u_{j}\left(\mathbf{x}^{\prime}\right) v_{j}^{*}(\mathbf{x})\right]\right\} .
\end{aligned}
$$

It is important to note here that we have ignored the interactions between the noncondensate particles in Eq. (9) because we are interested in temperatures much lower than condensation temperature and the depletion remains small.

The calculation of noncondensate densities in terms of the condensate wave function in principle requires the determination of the full spectrum of the BdG equations. While the first few modes of these equations would have wavelengths close to the size of the system, the wavelength of the following BdG modes would get much smaller than the size of the system, or the coherence length of the condensate. For such modes, the condensate density and the external trapping potential are slowly varying and Eq. (9) can be solved with a local density approximation akin to the Wenzel-Kramers-Brillouin approximation. Within this approximation, the noncondensate densities are locally determined in terms of the condensate wave function. The self-consistency requirement can be stated only in terms of the condensate wave function, which yields the generalized GP equation.

At zero temperature, the condensate wave function then satisfies

$$
\left[\left(-\frac{\hbar^{2} \nabla^{2}}{2 M}+U_{\mathrm{tr}}(\mathbf{x})-\mu\right)+\Phi_{H}(\mathbf{x})+\Omega_{n}(\mathbf{x})+\Omega_{m}(\mathbf{x})\right] \Psi(\mathbf{x})=0,
$$

where the Hartree potential is $\Phi_{H}(\mathbf{x})=\int d^{3} \mathbf{x}^{\prime} V_{\text {int }}(\mathbf{x}-$ $\left.\mathbf{x}^{\prime}\right)\left[\left|\Psi\left(\mathbf{x}^{\prime}\right)\right|^{2}+\tilde{n}\left(\mathbf{x}^{\prime}\right)\right]$. The local-fluctuation-induced potential, which is equivalent to the Lee-Huang-Yang (LHY) correction, has two contributions coming from direct and anomalous noncondensate densities,

$$
\begin{aligned}
& \Omega_{n}(\mathbf{x}) \Psi(\mathbf{x})=\frac{8}{3} g n_{0}(\mathbf{x}) \sqrt{\frac{a_{s}^{3} n_{0}(\mathbf{x})}{\pi}} \mathcal{Q}_{5}\left(\epsilon_{d d}\right) \Psi(\mathbf{x}), \\
& \Omega_{m}(\mathbf{x}) \Psi(\mathbf{x})=8 g n_{0}(\mathbf{x}) \sqrt{\frac{a_{s}^{3} n_{0}(\mathbf{x})}{\pi}} \mathcal{Q}_{5}\left(\epsilon_{d d}\right) \Psi(\mathbf{x}),
\end{aligned}
$$

where $Q_{l}\left(\epsilon_{d d}\right)=\int_{0}^{1} d u\left[1+\epsilon_{d d}\left(3 u^{2}-1\right)\right]^{l / 2}$. Explicitly, this is almost the generalized GP equation used in the literature $[11,14]$,

$$
\begin{aligned}
0= & {\left[-\frac{\hbar^{2} \nabla^{2}}{2 M}+U_{\mathrm{tr}}(\mathbf{x})-\mu+\int d^{3} \mathbf{x}^{\prime} V_{\mathrm{int}}\left(\mathbf{x}-\mathbf{x}^{\prime}\right)\left[\left|\Psi\left(\mathbf{x}^{\prime}\right)\right|^{2}\right.\right.} \\
& \left.\left.+\tilde{n}\left(\mathbf{x}^{\prime}\right)\right]+\frac{32}{3} g \sqrt{\frac{a_{s}^{3}}{\pi}} \mathcal{Q}_{5}\left(\epsilon_{d d}\right)|\Psi(\mathbf{x})|^{3}\right] \Psi(\mathbf{x})
\end{aligned}
$$

Thus the only correction to the generalized GP equation obtained by adding the local LHY correction to the chemical potential is the Hartree potential created by the depleted particles. If the depletion $\tilde{n}(\mathbf{x})=\frac{8}{3}\left(\frac{a_{s}^{3}}{\pi}\right)^{1 / 2} \mathcal{Q}_{3}\left(\epsilon_{d d}\right)|\Psi(\mathbf{x})|^{3}$ remains small, this contribution will be accordingly small.

If the dipolar interaction is not dominant, $\epsilon_{d d}<1$, the trapped gas will be stable at low densities and the fluctuation contribution is not qualitatively important. However, for strong dipolar interaction, $\epsilon_{d d}>1$, the gas has an instability towards collapse as the attractive part of the dipolar interaction can favor higher densities. The local-fluctuationinduced interaction potential can arrest this collapse because it scales with a higher power of the density, $n_{0}^{3 / 2}$, compared with the direct interaction with $n_{0}$. While this general physical mechanism has been tested experimentally [8] to be at play in the droplet experiments, the exact coefficient of the fluctuation term is not easy to determine. When $\epsilon_{d d}>1$ there is no stable solution for a uniform system, so some longwavelength modes will have imaginary frequencies, signaling collapse. Thus the expressions $\Omega_{n}$ and $\Omega_{m}$ [Eq. (12)] will have imaginary parts. One approach in the literature was to 
ignore the imaginary components, assuming they only cause decay at long times [9]. However, this imaginary part is clearly a by-product of the LDA, which, as we have discussed above, is valid only for higher Bogoliubov modes. A careful accounting of the discrete nature of the Bogoliubov modes would not show any imaginary frequencies, but a computationally much simpler approach is to impose a cutoff on which LDA modes should be taken into account, as employed in Refs. [11,14]. We used a spherical cutoff in $k$ space which excludes all modes with wave vectors smaller than $k_{c}=\frac{\pi}{2 \xi}$, as the coherence length of the condensate $\xi$ is the length scale which characterizes locality for the condensate.

The systematic derivation of the generalized GP equation using HFBT enables straightforward generalization to finite temperatures. While the HFBT is mostly reliable at all temperatures, local calculation of noncondensate densities solely in terms of local condensate wave function puts a severe restriction on the applicability of the LDA to obtain a generalized GP equation at nonzero temperature. The dynamics of condensate and noncondensate atoms are controlled by different equations, and only when the number of noncondensate atoms is small enough are their dynamics completely determined by the condensate. At zero temperature this essentially means that the system is sufficiently weakly interacting to limit quantum depletion. At finite temperature, noncondensate density is contributed to by both quantum and thermal depletion mechanisms. The noncondensate part of the dynamics will be controlled completely by the condensate only if the total depletion remains small. Essentially, to be able to write a single self-consistent equation for the condensate, the temperature must be much smaller than the BEC transition temperature so that the thermal depletion is comparable to the quantum depletion.

At such low but nonzero temperatures, the generalized GP equation takes the similar form:

$$
\left[-\frac{\hbar^{2} \nabla^{2}}{2 m}+U_{t r}(\mathbf{x})+\int d^{3} \mathbf{x}^{\prime} V_{\text {int }}\left(\mathbf{x}-\mathbf{x}^{\prime}\right)\left|\Psi\left(\mathbf{x}^{\prime}\right)\right|^{2}\right] \Psi(\mathbf{x})+\Delta \mu_{Q F}(\mathbf{x}, T) \Psi(\mathbf{x})=\mu \Psi(\mathbf{x}),
$$

with temperature only playing a role in the determination of the local-fluctuation-induced interaction. With the same cutoff used at zero temperature, we obtain

$$
\Delta \mu_{Q F}(\mathbf{x}, T)=\frac{32}{3} g \sqrt{\frac{a_{s}^{3}}{\pi}}\left[\mathcal{Q}_{5}\left(\epsilon_{d d}\right)+\mathcal{R}\left(\epsilon_{d d}, t(\mathbf{x})\right)\right]|\Psi(\mathbf{x})|^{3},
$$

with dimensionless temperature $t=k_{B} T / g n_{0}(\mathbf{x})$ and

$$
\begin{aligned}
\mathcal{Q}_{5}\left(\epsilon_{d d} ; q_{c}\right) & =\frac{1}{4 \sqrt{2}} \int_{0}^{1} d u f(u)\left\{\left[4 f(u)-q_{c}^{2}\right] \sqrt{2 f(u)+q_{c}^{2}}-3 f(u) q_{c}+q_{c}^{3}\right\}, \\
\mathcal{R}\left(\epsilon_{d d}, t ; q_{c}\right) & =\frac{3}{4 \sqrt{2}} \int_{0}^{1} d u \int_{q_{c}^{2}}^{\infty} d Q \frac{Q f(u)}{\sqrt{Q+2 f(u)}} \frac{1}{\exp [\sqrt{Q[Q+2 f(u)]} / t]-1} .
\end{aligned}
$$

In Ref. [16] we calculated these dimensionless functions and obtained a $t^{2}$ power-law fit $\mathcal{R}\left(\epsilon_{d d}, t\right)=S\left(\epsilon_{d d}\right) t^{2}$, and the coefficient is best approximated by $\mathcal{S}\left(\epsilon_{d d}\right)=-0.01029 \epsilon_{d d}^{4}+0.02963 \epsilon_{d d}^{3}-0.05422 \epsilon_{d d}^{2}+0.009302 \epsilon_{d d}+0.1698$ for $0<\epsilon_{d d}<2$.

\section{NUMERICAL CALCULATION OF THE DENSITY PROFILES}

We now discuss the numerical solution of Eq. (14) with the parametrization in the previous section and compare it with the variational solution presented in Ref. [16]. Our focus is on the observable effects of temperature on the density profile. As our LDA approach neglects the effect of interactions of the noncondensate atoms, the temperature range will be limited so as to make sure that the condensate fraction remains close to one.

We use the time-splitting spectral method [19] to evolve the GP equation in imaginary time until the convergence in energy is reached. A three-dimensional grid of up to 64 by 64 by 256 points in real space is used to capture the anisotropic shape of the droplets, where the real-space discretization step is determined to ensure smooth variation of the cloud density. The Hartree potential created by the long-range dipolar interaction is calculated by expressing it as a convolution in $k$ space:

$$
V_{H}^{(d d)}(\mathbf{x})=\int \frac{d^{3} \mathbf{k}}{(2 \pi)^{3}} \tilde{n}_{0}(\mathbf{k}) \tilde{V}_{d d}(\mathbf{k}) e^{i \mathbf{k} \cdot \mathbf{x}}
$$

where the Fourier transform of the dipolar interaction is

$$
\tilde{V}_{d d}(\mathbf{k})=\epsilon_{d d}\left(3 \frac{k_{z}^{2}}{|\mathbf{k}|^{2}}-1\right)
$$

While this expression avoids the complications of the dipolar potential near zero separation, the use of discrete Fourier transforms instead of the continuum integrals effectively introduces repeated copies of the simulation cell. To avoid the interaction between neighboring real-space cells, we used a spherical cutoff on the dipolar interaction which sets the interaction to zero beyond the cutoff distance. This cutoff distance must be chosen larger than the maximum size of the droplet so that the points at the edges of the cigar-shaped cloud interact with each other. However, it must be shorter than then the distance between the closest points of clouds in spuriously repeated simulation cells, so that long-range interactions take place only within one simulation cell. We choose the size of the simulation cell and the cutoff to comply with these constraints. The Fourier transform of the dipolar potential 
with this cutoff is calculated as

$$
\tilde{V}_{d d}^{\text {(cut) }}(\mathbf{k})=\tilde{V}_{d d}(\mathbf{k})\left(1-3 \frac{\sin (|\mathbf{k}| R)-|\mathbf{k}| R \cos (|\mathbf{k}| R)}{(|\mathbf{k}| R)^{3}}\right) .
$$

After numerically obtaining the solution, we extract the widths by using the moments such that they correspond to the variational width parameters when applied to a variational Gaussian state [13],

$$
\begin{aligned}
\sigma_{\rho}^{2} & =\frac{4}{N} \int d^{3} \mathbf{x} \rho^{2}|\Psi(\mathbf{x})|^{2}, \\
\sigma_{z}^{2} & =\frac{2}{N} \int d^{3} \mathbf{x} z^{2}|\Psi(\mathbf{x})|^{2} .
\end{aligned}
$$

We first consider a parameter regime for which there is a sharp transition from a pancake-shaped low-density cloud to a single droplet. Focusing on the parameter regime explored in the Stuttgart experiments [7-9], we calculate the density profile for $N=2000$ Dy atoms in an axially symmetric trap with frequencies $f_{\perp}=45 \mathrm{~Hz}, f_{z}=133 \mathrm{~Hz}$. At zero temperature the cloud enters the droplet state below $a_{c}^{(0)}=83 a_{0}$. As can be seen in Fig. 1, increasing temperature shifts this transition to longer scattering lengths, where a $100 \mathrm{nK}$ change in the cloud temperature can be observed to make a $\approx 10 a_{0}$ change in the transition point. It is possible to induce the transition from the cloud to the droplet by increasing the temperature, as shown in Fig. 1, for $a_{s}=88 a_{0}$ the cloud enters the droplet state around $T \simeq 110 \mathrm{nK}$.

We see from our derivation that there are three effects of raising the temperature in a dipolar droplet system. The first effect will be that the LHY term increases as thermal fluctuations now supplement quantum fluctuations stabilizing the droplet state. The second effect is that the number of particles in the condensate is lowered because of the thermal depletion, and, following this, the third effect is that the depleted particle density will have long-range dipolar interactions with the condensate. Our calculations show that, at the lowest temperatures which are relevant for the current experiments, the change in the compressibility of the droplet, i.e., the LHY term, will be the most dominant of these three effects. However, even though the temperature contribution to LHY term is always positive, its effect on the transition between the low density and the droplet state is nontrivial.

Let us particularly focus on the pancake-shaped traps for which the distinction between the droplet and low-density phases is sharp. The attractive part of the dipolar interaction favors higher density and higher aspect ratios for the cloud, which would normally collapse the cloud for $\epsilon_{D D}>1$. This inclination is resisted by two different mechanisms for the low-density and droplet phases. In the low-density phase the trap potential along the $z$ direction combined with the short-range repulsion stops the collapse. The effect of low temperature is negligible for the low-density minimum. For the droplet phase the confining potential is irrelevant and the collapse is arrested by the LHY energy which increases with a higher power of density. The first effect of temperature is an increase in the coefficient of the LHY term.

In a pancake trap, the transition is first order, so the system chooses the local minimum with the lower energy. As temperature is increased the minimum energy corresponding
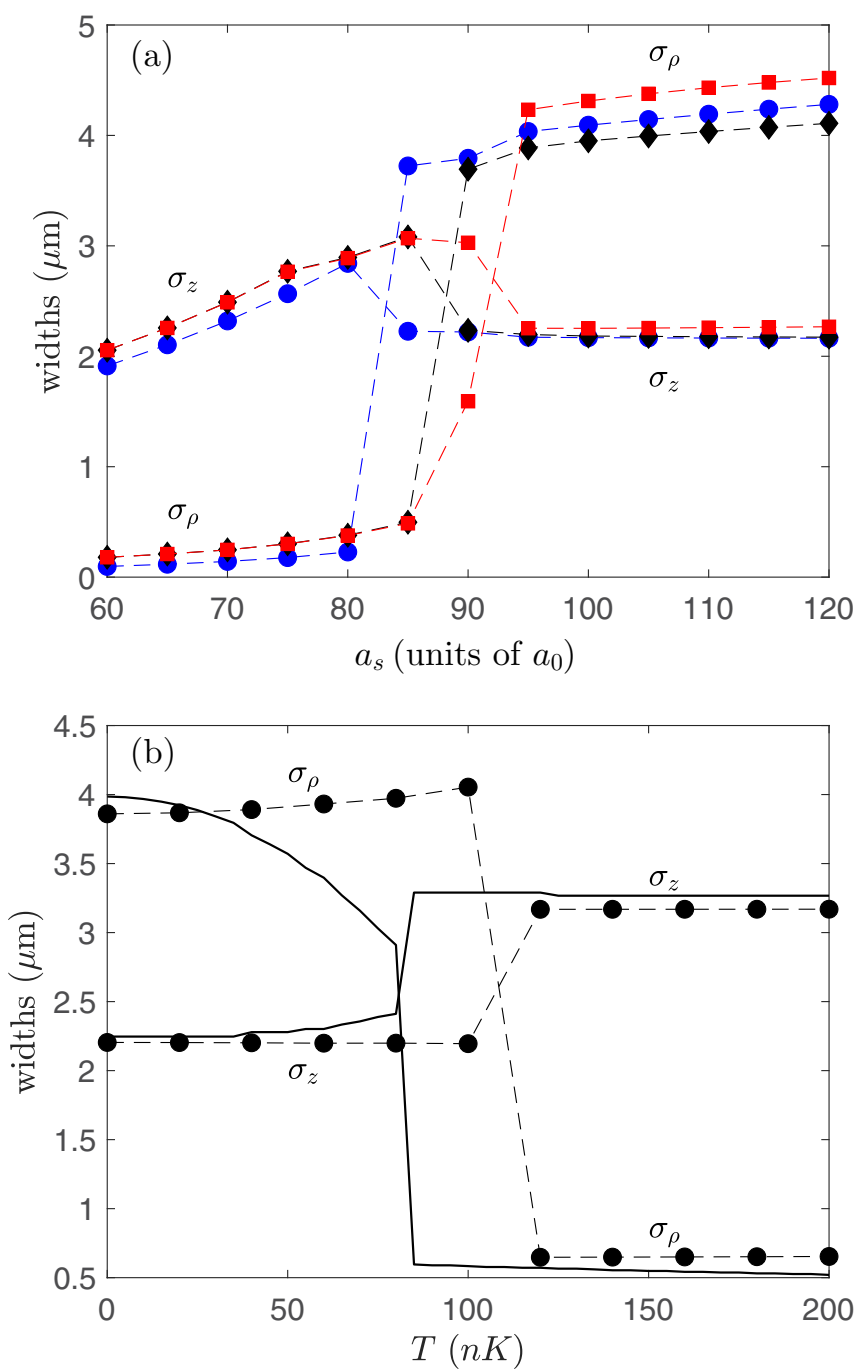

FIG. 1. (a) Radial and axial widths, $\sigma_{\rho}$ and $\sigma_{z}$, of a Dy BEC with $N=2000$ atoms in a pancake-shaped trap with harmonic frequencies $\left\{\omega_{\rho}, \omega_{z}\right\}=2 \pi \times\{45,133\} \mathrm{Hz}$ as a function of the $s$-wave scattering length $a_{s}$. The dots, diamonds, and squares correspond to the temperatures $T=0, T=70 \mathrm{nK}$, and $T=150 \mathrm{nK}$, respectively. The dashed lines are guides for the eye and the points are obtained by numerical time evolution. The cloud density and the transition point to a high-density droplet phase is significantly altered as the temperature is varied. At a critical $a_{s}$ value of $88 a_{0}$, panel (b) shows the change of radial and axial widths as a function of the temperature. The dots show the results of numerical time evolution and the solid line is obtained by assuming a Gaussian ansatz.

to the low-density phase barely changes, while the minimum energy for the droplet changes due to the change in the LHY term. While the LHY contribution to energy increases, the droplet aspect ratio and thus the negative contribution of the dipolar interaction energy also increases. Increasing temperature lowers the total energy of the droplet minimum, resulting in the transition to the droplet state seen in Fig. 1. While we base this argument on the variational study in Ref. [16], numerical results obtained here show that the nontrivial effect of the temperature on the transition is not an artifact of the restrictions to Gaussian wave functions. The results of the numerical calculation are in good agreement with the variational 


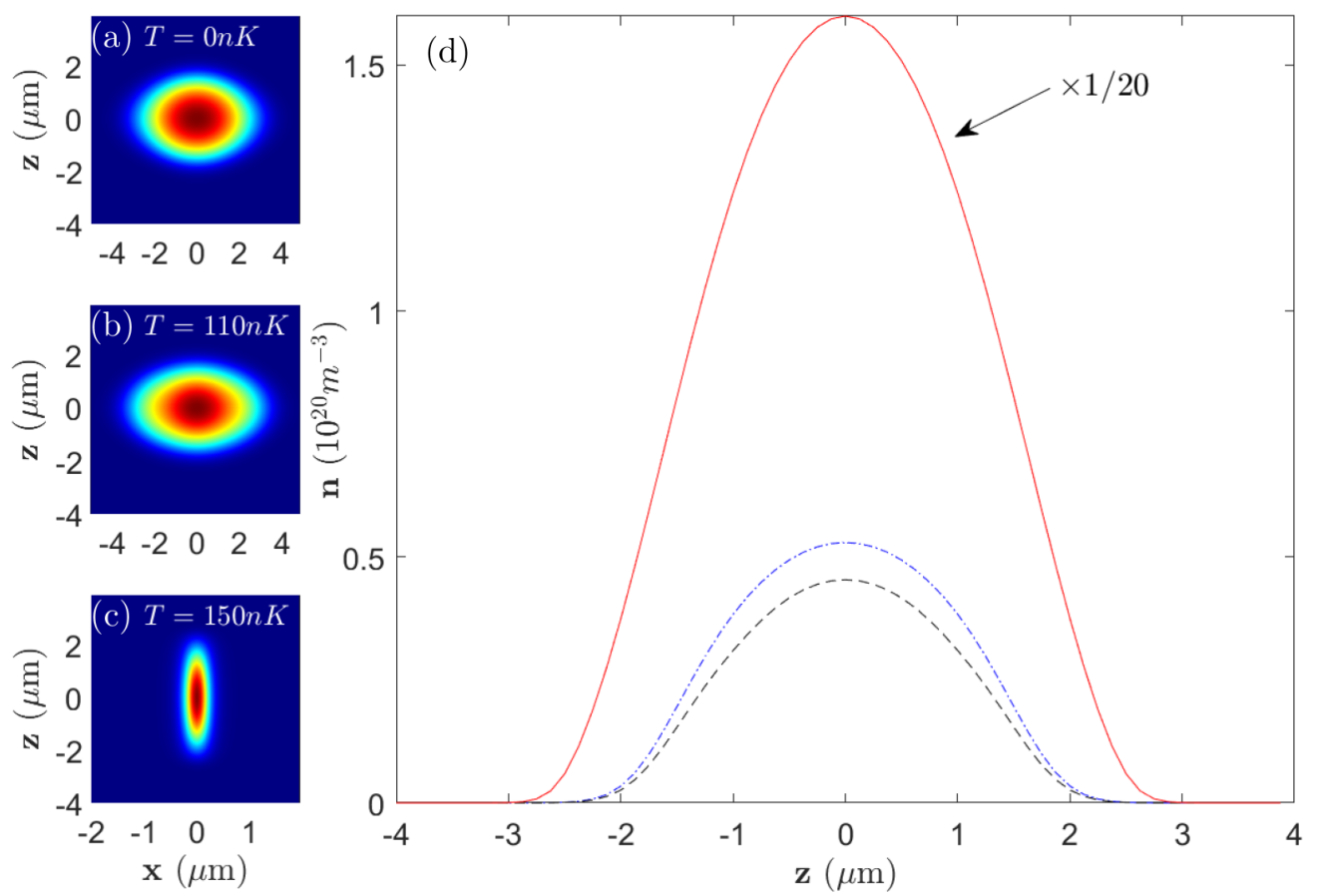

FIG. 2. (a)-(c) Density profile $(x, z, y=0)$ of a Dy BEC with $N=2000$ atoms and $a_{s}=88$ at $T=0, T=110 \mathrm{nK}, T=150 \mathrm{nK}$, respectively, in a pancake-shaped trap with harmonic frequencies $\left\{\omega_{\rho}, \omega_{z}\right\}=2 \pi \times\{45,133\} \mathrm{Hz}$. The $2 \mathrm{D}$ profiles show the transition from a BEC to a high-density droplet as the temperature is increased. Panel (d) shows the cuts along the $z$ direction with $x, y=0$. The blue (dash-dotted), black (dashed), and red (solid) lines correspond to the temperatures $T=0, T=110 \mathrm{nK}$, and $T=150 \mathrm{nK}$, respectively. For visual clarity the density cut for the $T=150 \mathrm{nK}$ case (red) is reduced by a factor of 20 .

calculation presented in Ref. [16]. As can be seen in Fig. 2, even though the density of the center changes by a factor of $\simeq 20$, the profile of the cloud remains well approximated by a Gaussian throughout the transition, thus the variational fits retain their predictive power.

We also simulate what happens in the deep droplet state as can be seen in Fig. 3, the density profile is not a Gaussian any more, and the numerical solution is different from the variational result. The effect of low temperatures on this strong droplet state remains negligible.

Next, we investigate a parameter regime for which the transition between the droplet and low-density phases is smooth, as observed in the Innsbruck experiments [10]. For $20000 \mathrm{Er}$ atoms in a trap with frequencies $f_{\perp}=178 \mathrm{~Hz}, f_{z}=17.2 \mathrm{~Hz}$
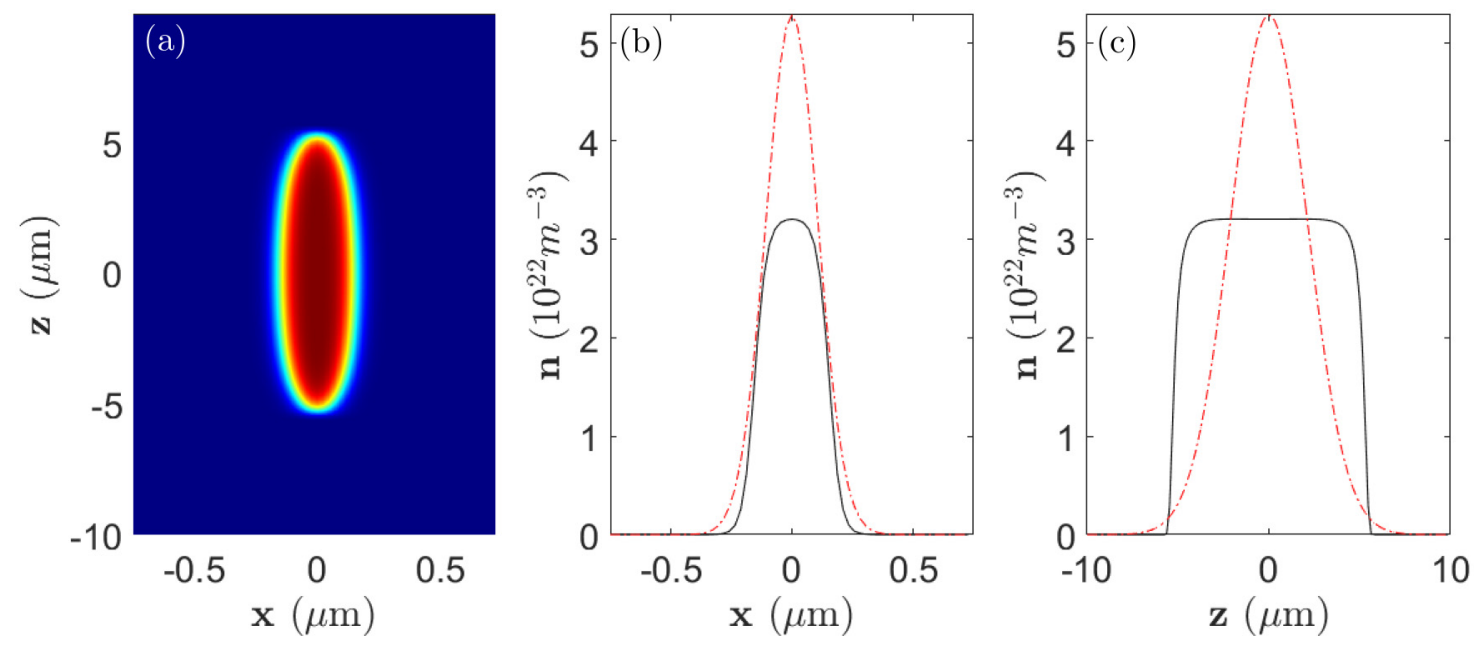

FIG. 3. (a) Density profile $(x, z, y=0)$ of a high-density Dy droplet with $N=20000$ atoms and $a_{s}=60$ in a spherical trap with $v_{i}=70 \mathrm{~Hz}$ at $T=0$ obtained by imaginary time evolution. Panels (b) and (c) show the cuts along the $x$ and $z$ directions with $y, z=0$ and $x, y=0$, respectively. The black (solid) lines are obtained by imaginary time evolution and the flat-top profile of the high-density droplets is apparent. The red (dash-dotted) lines show the analytical results assuming a Gaussian ansatz. The difference between the ground-state density profiles manifests itself in the lowest-lying excitations. The axial oscillation frequencies for the exact numerical density profile and the Gaussian ansatz are $5.01 v_{z}$ and $3.96 v_{z}$, respectively, where $v_{z}$ is the axial trap frequency. 


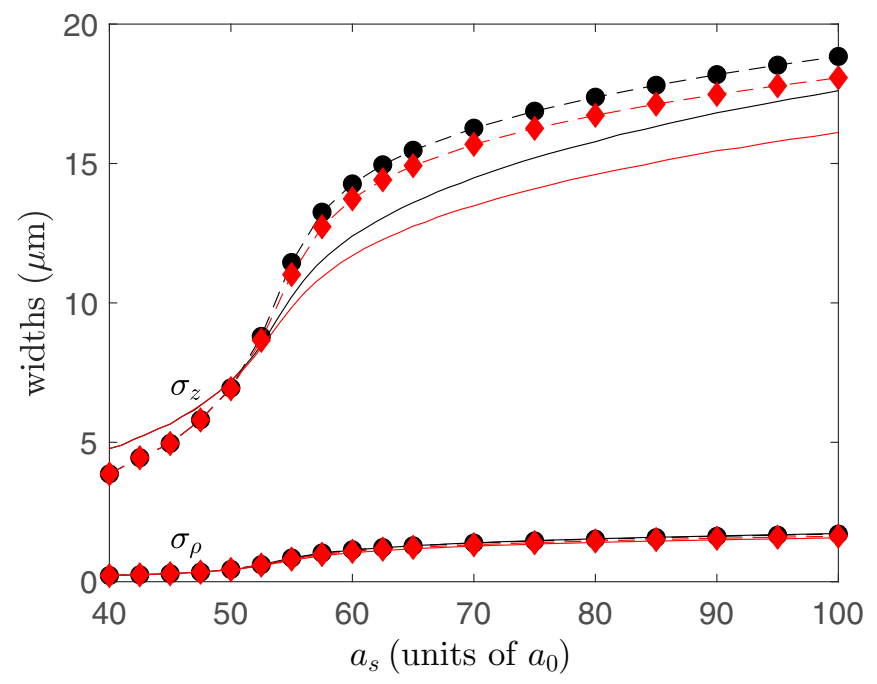

FIG. 4. (a) Radial and axial widths, $\sigma_{\rho}$ and $\sigma_{z}$, of an Er BEC with $N=20000$ atoms in a cigar-shaped trap with harmonic frequencies $\left\{\omega_{\rho}, \omega_{z}\right\}=2 \pi \times\{178,17\} \mathrm{Hz}$ as a function of the $s$-wave scattering length $a_{s}$. The black line and dots correspond to the temperature $T=$ 0 , the red line and diamonds correspond to $T=150 \mathrm{nK}$. The dashed lines in the figure are guides for the eye. The solid lines are obtained analytically by assuming a Gaussian ansatz, and the points are the results of the numerical time evolution. The finite-temperature effects are observable yet not significant for the cigar-shaped droplets.

the transition to the droplet is a continuous crossover near $a_{s} \simeq 50 a_{0}$. We find that the temperatures up to $200 \mathrm{nK}$ have a distinct, yet small, effect on the cloud radii throughout, as can be seen in Fig. 4. Once again the density profile of the cloud remains Gaussian throughout the transition and the variational results are in good agreement with the numerical calculations.

\section{OSCILLATION FREQUENCIES OF DROPLETS AT FINITE TEMPERATURE}

Collective oscillations of trapped condensates have emerged as one of the most valuable experimental probes from the earliest experiments on ultracold atoms. Similarly, thermal activation of collective modes for superfluid helium droplets were investigated [20]. The collective excitation frequencies of a trapped cloud reveal information about the equilibrium state and are, in general, sensitive to the local compressibility of the system. For a short-range interacting BEC, the collective oscillation frequencies are only weakly dependent on temperature, except near the BEC critical temperature [21]. However, the dipolar droplet state is stabilized by fluctuations and the equilibrium can be more sensitive to thermal effects.

The oscillation modes for a dipolar gas at zero temperature has been calculated in Ref. [22]. For the droplet state the lowest-lying oscillation modes have been calculated in Ref. [12] by a variational approach, while the more general spectrum of excited modes of the droplet have been explored numerically by Ref. [15]. The oscillation frequencies have been measured for Er droplets [10], where the signature of the local LHY correction was clearly identified in the axial oscillation mode frequency. Similarly, collective oscillations in Dy droplets have been excited by tilting the dipole orientations [23].

We explore the few lowest-lying modes of the droplet through both approaches. First, we write a variational Lagrangian for a Gaussian wave function for which coupled oscillations of the cloud width in three dimensions give us the lowest three modes of the condensate. We compare the values found from this approach with the numerical real-time evolution of the condensate.

We use a Gaussian ansatz for the wave function,

$$
\begin{aligned}
\Psi(x, y, z, t)= & \frac{\sqrt{N}}{\pi^{3 / 4}\left[w_{x}(t) w_{y}(t) w_{z}(t)\right]^{1 / 2}} \\
& \times \exp \left\{-\frac{1}{2}\left(\frac{x^{2}}{w_{x}(t)^{2}}+\frac{y^{2}}{w_{y}(t)^{2}}+\frac{z^{2}}{w_{z}(t)^{2}}\right)\right. \\
& \left.+i\left[x^{2} \beta_{x}(t)+y^{2} \beta_{y}(t)+z^{2} \beta_{z}(t)\right]\right\}
\end{aligned}
$$

where the time-dependent parameters $w_{x}, w_{y}$, and $w_{z}$ give the cloud radii. The other variational parameters $\beta_{x}, \beta_{y}$, and $\beta_{z}$ facilitate the mass current so that the cloud density can oscillate. Such a restricted form for the variational wave function can only capture the three collective oscillation frequencies of the condensate. However, these lowest modes have been the most accessible modes experimentally and variational Lagrangian method results have been in good agreement with measurements $[10,12]$.

For elongated cigar-shaped condensates such as the dipolar droplets, one of these modes feature oscillations predominantly in the axial direction. This axial mode has the lowest frequency which has been measured in Er and Dy experiments. The other two modes consist of breathing and quadrupolar excitations perpendicular to the long axis and typically have frequencies an order of magnitude larger than the axial mode for the droplets.

The effect of the beyond-mean-field correction for the oscillation frequencies were first reported in Ref. [12]. We now introduce the temperature dependence into the LHY correction. Our Lagrangian is

$$
\begin{aligned}
\mathcal{L}= & \frac{i \hbar}{2}\left(\Psi(\mathbf{x}, t) \frac{\partial \Psi^{*}(\mathbf{x}, t)}{\partial t}-\Psi^{*}(\mathbf{x}, t) \frac{\partial \Psi(\mathbf{x}, t)}{\partial t}\right) \\
& +\frac{\hbar^{2}}{2 M}|\nabla \Psi(\mathbf{x}, t)|^{2}+U_{\mathrm{tr}}(\mathbf{x})|\Psi(\mathbf{x}, t)|^{2} \\
& +\frac{1}{2} \int d^{3} \mathbf{x}^{\prime}|\Psi(\mathbf{x}, t)|^{2} V_{\mathrm{int}}\left(\mathbf{x}-\mathbf{x}^{\prime}\right)\left|\Psi\left(\mathbf{x}^{\prime}, t\right)\right|^{2} \\
& +\frac{2}{5} \gamma|\Psi(\mathbf{x}, t)|^{5}+2 \theta T^{2}|\Psi(\mathbf{x}, t)|,
\end{aligned}
$$

where $\gamma=\frac{32}{3} g\left(\frac{a_{s}^{3}}{\pi}\right)^{1 / 2} \mathcal{Q}_{5}\left(\epsilon_{d d}\right)$, and $\theta=\frac{32}{3} g\left(\frac{a_{s}^{3}}{\pi}\right)^{1 / 2} \frac{k_{B}^{2}}{g^{2}} \mathcal{S}\left(\epsilon_{d d}\right)$.

The integrals in the Lagrangian can be carried out for the variational wave function to yield the 
Lagrangian in terms of the variational parameters as

$$
\begin{aligned}
L\left(w_{x}, w_{y}, w_{z}, \beta_{x}, \beta_{y}, \beta_{z}\right)= & \frac{N \hbar}{2}\left(w_{x}^{2} \dot{\beta}_{x}+w_{y}^{2} \dot{\beta}_{y}+w_{z}^{2} \dot{\beta}_{z}\right)+\frac{\hbar^{2} N}{4 M}\left(\frac{1}{w_{x}^{2}}+\frac{1}{w_{y}^{2}}+\frac{1}{w_{z}^{2}}+4 w_{x}^{2} \beta_{x}^{2}+4 w_{y}^{2} \beta_{y}^{2}+4 w_{z}^{2} \beta_{z}^{2}\right) \\
& +\frac{1}{4} M N\left(\omega_{x}^{2} w_{x}^{2}+\omega_{y}^{2} w_{y}^{2}+\omega_{z}^{2} w_{z}^{2}\right)+\frac{g N^{2}}{2} \frac{1}{2^{3 / 2} \pi^{3 / 2} w_{x} w_{y} w_{z}}\left[1-\epsilon_{d d} F\left(\frac{w_{x}}{w_{z}}, \frac{w_{y}}{w_{z}}\right)\right] \\
& +\frac{4 \sqrt{2}}{25 \sqrt{5} \pi^{9 / 4}} \frac{N^{5 / 2} \gamma}{\left(w_{x} w_{y} w_{z}\right)^{3 / 2}}+4 \sqrt{2} \pi^{3 / 4} \theta T^{2} N^{1 / 2}\left(w_{x} w_{y} w_{z}\right)^{1 / 2},
\end{aligned}
$$

where

$$
F\left(\kappa_{x}, \kappa_{y}\right)=1-3 \kappa_{x} \kappa_{y} \int_{0}^{\pi} \frac{d \phi}{\pi}\left(\frac{\tanh ^{-1}\left[\sqrt{1-\left(\kappa_{x}^{2} \cos ^{2} \phi+\kappa_{y}^{2} \sin ^{2} \phi\right)}\right]}{\left[1-\left(\kappa_{x}^{2} \cos ^{2} \phi+\kappa_{y}^{2} \sin ^{2} \phi\right)\right]^{3 / 2}}-\frac{1}{1-\left(\kappa_{x}^{2} \cos ^{2} \phi+\kappa_{y}^{2} \sin ^{2} \phi\right)}\right) .
$$

The Euler-Lagrange equations for the radii give

$$
\hbar w_{\eta} \dot{\beta}_{\eta}+\frac{2 \hbar^{2}}{M} w_{\eta} \beta_{\eta}^{2}+\frac{\partial G}{\partial w_{\eta}}=0,
$$

while the phase terms are simply related to the velocity of the radii as

$$
\beta_{\eta}=\frac{M}{2 \hbar w_{\eta}} \dot{w}_{\eta},
$$

where,

$$
\begin{aligned}
G\left(w_{x}, w_{y}, w_{z}\right)= & \frac{\hbar^{2}}{4 M}\left(\frac{1}{w_{x}^{2}}+\frac{1}{w_{y}^{2}}+\frac{1}{w_{z}^{2}}\right)+\frac{1}{4} M\left(\omega_{x}^{2} w_{x}^{2}+\omega_{y}^{2} w_{y}^{2}+\omega_{z}^{2} w_{z}^{2}\right)+\frac{g N}{2} \frac{1}{2^{3 / 2} \pi^{3 / 2} w_{x} w_{y} w_{z}}\left[1-\epsilon_{d d} F\left(\frac{w_{x}}{w_{z}}, \frac{w_{y}}{w_{z}}\right)\right] \\
& +\frac{4 \sqrt{2}}{25 \sqrt{5} \pi^{9 / 4}} \frac{N^{3 / 2} \gamma}{\left(w_{x} w_{y} w_{z}\right)^{3 / 2}}+4 \sqrt{2} \pi^{3 / 4} \theta T^{2} N^{-1 / 2}\left(w_{x} w_{y} w_{z}\right)^{1 / 2}
\end{aligned}
$$

Therefore,

$$
\frac{d^{2} w_{\eta}}{d t^{2}}=-\frac{2}{M} \frac{\partial}{\partial w_{\eta}} G\left(w_{x}, w_{y}, w_{z}\right) .
$$

We nondimensionalize the equations in terms of the effective trap frequency $\bar{\omega}=\left(\omega_{x} \omega_{y} \omega_{z}\right)^{1 / 3}$, and the harmonic-oscillator length $\bar{l}=\sqrt{\frac{\hbar}{M \bar{\omega}}}$. With $v_{i}=w_{i} / \bar{l}, \tau=\bar{\omega} t$, and $E_{0}=\hbar \bar{\omega}$, the effective potential $\tilde{U}$ becomes

$$
\begin{aligned}
\tilde{U}= & \frac{1}{2}\left(\frac{1}{v_{x}^{2}}+\frac{1}{v_{y}^{2}}+\frac{1}{v_{z}^{2}}\right)+\frac{1}{2}\left(\frac{\omega_{x}^{2}}{\bar{\omega}^{2}} v_{x}^{2}+\frac{\omega_{y}^{2}}{\bar{\omega}^{2}} v_{y}^{2}+\frac{\omega_{z}^{2}}{\bar{\omega}^{2}} v_{z}^{2}\right)+\sqrt{\frac{2}{\pi}} \frac{N a_{s}}{\bar{l}} \frac{1}{v_{x} v_{y} \nu_{z}}\left[1-\epsilon_{d d} F\left(\frac{v_{x}}{v_{z}}, \frac{v_{y}}{v_{z}}\right)\right] \\
& +\mathbf{c}_{1} \frac{N^{3 / 2} a_{s}^{5 / 2}}{\bar{l}^{5 / 2}} \frac{1}{\left(v_{x} v_{y} v_{z}\right)^{3 / 2}} \mathcal{Q}_{5}\left(\epsilon_{d d}\right)+\mathbf{c}_{2} \frac{a_{s}^{1 / 2}}{N^{1 / 2} \bar{l}^{1 / 2}}\left(v_{x} v_{y} v_{z}\right)^{1 / 2} \tilde{t}^{2} \mathcal{S}\left(\epsilon_{d d}\right),
\end{aligned}
$$

where $\mathbf{c}_{1}=\frac{1024 \sqrt{2}}{75 \sqrt{5} \pi^{7 / 4}} \approx 1.1648$, and $\mathbf{c}_{2}=\frac{64 \sqrt{2}}{3 \pi^{3 / 4}} \approx 12.785$. The equilibrium radii are found at the stationary point of the effective potential

$$
\left.\frac{\partial \tilde{U}}{\partial v_{\eta}}\right|_{v_{\eta(0)}}=0
$$

Taylor expansion to second order near the equilibrium point defines the Hessian matrix

$$
M=\left[\begin{array}{ccc}
k_{x} & \lambda_{x y} & \lambda_{z x} \\
\lambda_{x y} & k_{y} & \lambda_{y z} \\
\lambda_{z x} & \lambda_{y z} & k_{z}
\end{array}\right]
$$

where $k_{\eta}=\left.\frac{\partial^{2} \tilde{U}}{\partial v_{\eta}^{2}}\right|_{(0)}, \lambda_{\alpha, \beta}=\left.\frac{\partial^{2} \tilde{U}}{\partial v_{\alpha} \partial v_{\beta}}\right|_{(0)}$. Collective frequencies are found via diagonalization of this matrix. We numerically calculate the derivatives near the equilibrium point and obtain the collective oscillation frequencies.

As a check on our variational results we also calculate the oscillation frequencies numerically through real-time evolution with the split step method outlined in the previous section. We first calculate the oscillation frequencies for the Dy experimental parameters for which there is an abrupt transition. The oscillation frequencies increase upon transition to the droplet state, as can be seen in Fig. 5. For 20000 Dy atoms in a spherical trap with $f=75 \mathrm{~Hz}$ the 


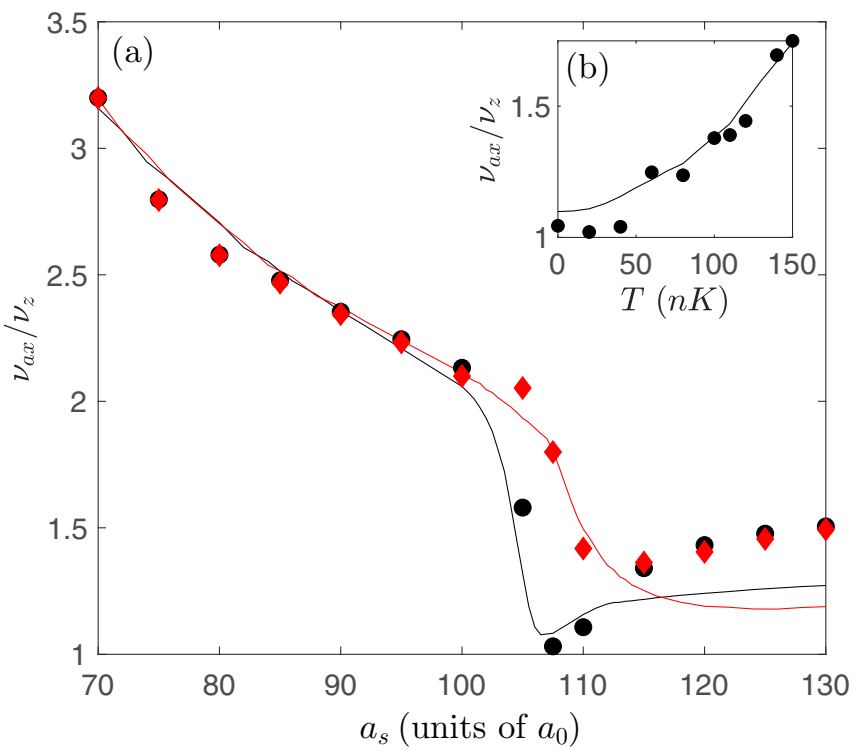

FIG. 5. (a) Axial oscillation frequencies of a Dy BEC with $N=$ 2000 atoms in a pancake-shaped trap with harmonic frequencies $\left\{\omega_{\rho}, \omega_{z}\right\}=2 \pi \times\{70,70\} \mathrm{Hz}$ as a function of the $s$-wave scattering length $a_{s}$. The black (lower) line and dots correspond to the temperature $T=0$, the red (upper) line and diamonds correspond to $T=150 \mathrm{nK}$. The lines are obtained analytically by assuming a Gaussian ansatz, and the points are the results of numerical time evolution. Around the transition point to a high-density droplet, the effect of temperature on the axial oscillations is significant and the frequencies are altered by around $75 \%$. At a critical $a_{s}$ value of $108 a_{0}$, panel (b) shows the change of the axial oscillation frequency as a function of temperature. Both figures show the frequencies in units of the axial trap frequency.

transition takes place near $a_{s} \simeq 105 a_{0}$ and is signalled by an abrupt change in the axial oscillation frequency. Repeating the same calculation for $T=150 \mathrm{nK}$ we observe that the transition in the frequency becomes smoother as the transition point moves to higher $a_{s}$. Thus, the most dramatic effect of temperature on the oscillation frequencies happens near the transition. The change in the frequency of the axial mode as a function of temperature is displayed in Fig. 5, where we observe close to $70 \%$ change near the critical $a_{s}=105 a_{0}$. Both variational and numerical results give parallel physical pictures, indicating that the increase in the oscillation frequency with temperature is greatest near the transition to the droplet state.

In the Er experiments the cigar-shaped condensate has a smooth transition between the low-density and droplet states. This smooth transition causes the cloud shape to be mostly immune to changes in temperature, as explored in the previous section. Both the time-dependent variational approach and the numerical real-time evolution predict a sharp rise in the axial mode frequency upon droplet formation, which agree with the experimental observations in Ref. [10]. The variational approach predicts an increase in the oscillation frequencies with temperature, as can be seen in Fig. 6. However, numerical solutions of the same equation show very little change from the zero-temperature frequencies up to $150 \mathrm{nK}$. As the droplet state is extremely elongated, the variational approach may be

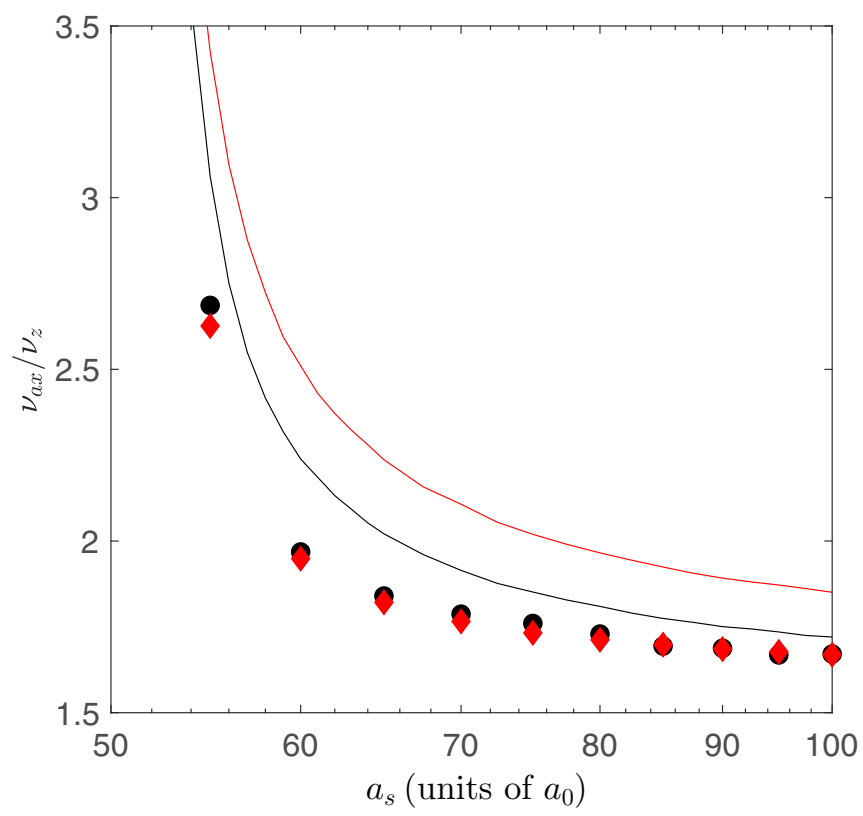

FIG. 6. Axial oscillation frequencies of an Er BEC with $N=$ 20000 atoms in a cigar-shaped trap with harmonic frequencies $\left\{\omega_{\rho}, \omega_{z}\right\}=2 \pi \times\{178,17\} \mathrm{Hz}$ as a function of the $s$-wave scattering length $a_{s}$. The oscillation frequency is in the units of axial trap frequency. The black (lower) line and dots correspond to the temperature $T=0$, the red (upper) line and diamonds correspond to $T=150 \mathrm{nK}$. The lines are obtained analytically by assuming a Gaussian ansatz and the dots are the results of numerical time evolution. For the cigar-shaped droplets the low-energy excitations are not altered significantly by the finite-temperature effects.

overstating the importance of the compressibility of the center of the cloud by disregarding higher modes along the axial direction. Thus, we expect no significant low-temperature effect on the axial oscillation frequency close to smooth crossover to the droplet state.

\section{RELEVANCE FOR RECENT EXPERIMENTS}

In this paper we provide further support for the claim that dipolar droplets are particularly susceptible to thermal fluctuations at low temperatures. Numerical calculations presented here support the conclusions of the previous variational calculation [16]. Furthermore, we show that the oscillation frequencies of collective modes are also sensitive to the temperature near the droplet transition. This should be expected because the collective modes are good probes of compressibility and the primary effect of temperature is to change the droplet's local chemical potential.

Standard experimental methods for thermometry are not applicable to the current droplet experiments. The small size of the droplets impede the in situ density measurement of the thermal component. Long-range dipolar interactions affect the free expansion of the condensate, even when the droplet is not self-trapped [24]. The temperature data given in the literature are based on the thermal component before droplet formation for both the Dy [7] and the Er systems [10]. In the absence of a reliable temperature measurement, it is not possible to make a direct comparison of our results with the 
experimental data, either for the Dy or Er experiments. Our results show that a change in the oscillation frequencies can occur even at temperatures much lower than the BEC transition temperature, and this may explain the uncertainty in the range of frequencies observed in Refs. [10,23]. A change in the oscillation frequency for systems intentionally prepared at a higher temperature, or heated after droplet formation by an external probe, would provide convincing evidence for the role of thermal fluctuations on dipolar droplets.

It is important to list the limitations of the approach used here. Our theory does not take the interactions between excited (above the condensate) particles into account, which limits its applicability to systems where the number of thermal atoms is close to the number of atoms in the condensate. Fluctuations are treated in the local density approximation, hence the independent dynamics of the thermal cloud is not considered. Furthermore, the expression for the local chemical potential suffers from an ad hoc cutoff. These limitations make it hard to propose the collective oscillation frequencies as a quantitative measure of temperature. However, we believe that the dependence of the oscillation frequencies on temperature should be observable in the current experimental regime.

\section{ACKNOWLEDGMENT}

This project was supported by Türkiye Bilimsel ve Teknolojik Araştırma Kurumu (TÜBİTAK) Grant No. $116 \mathrm{~F} 215$.
[1] L. P. Pitaevskii, Sov. Phys. JETP 13, 451 (1961).

[2] E. P. Gross, Structure of a quantized vortex in boson systems, Nuovo Cimento 20, 454 (1961).

[3] D. S. Petrov, Quantum Mechanical Stabilization of a Collapsing Bose-Bose Mixture, Phys. Rev. Lett. 115, 155302 (2015).

[4] C. R. Cabrera, L. Tanzi, J. Sanz, B. Naylor, P. Thomas, P. Cheiney, and L. Tarruell, Quantum liquid droplets in a mixture of Bose-Einstein condensates, Science 359, 301 (2018).

[5] G. Semeghini, G. Ferioli, L. Masi, C. Mazzinghi, L. Wolswijk, F. Minardi, M. Modugno, G. Modugno, M. Inguscio, and M. Fattori, Self-Bound Quantum Droplets of Atomic Mixtures in Free Space, Phys. Rev. Lett. 120, 235301 (2018).

[6] C. D’Errico, A. Burchianti, M. Prevedelli, L. Salasnich, F. Ancilotto, M. Modugno, F. Minardi, and C. Fort, Observation of quantum droplets in a heteronuclear bosonic mixture, Phys. Rev. Res. 1, 033155 (2019).

[7] H. Kadau, M. Schmitt, M. Wenzel, C. Wink, T. Maier, I. Ferrier-Barbut, and T. Pfau, Observing the Rosensweig instability of a quantum ferrofluid, Nature (London) 530, 194 (2016).

[8] I. Ferrier-Barbut, H. Kadau, M. Schmitt, M. Wenzel, and T. Pfau, Observation of Quantum Droplets in a Strongly Dipolar Bose Gas, Phys. Rev. Lett. 116, 215301 (2016).

[9] M. Schmitt, M. Wenzel, F. Böttcher, I. Ferrier-Barbut, and T. Pfau, Self-bound droplets of a dilute magnetic quantum liquid, Nature (London) 539, 259 (2016).

[10] L. Chomaz, S. Baier, D. Petter, M. J. Mark, F. Wächtler, L. Santos, and F. Ferlaino, Quantum-Fluctuation-Driven Crossover from a Dilute Bose-Einstein Condensate to a Macrodroplet in a Dipolar Quantum Fluid, Phys. Rev. X 6, 041039 (2016).

[11] F. Wächtler and L. Santos, Quantum filaments in dipolar BoseEinstein condensates, Phys. Rev. A 93, 061603(R) (2016).

[12] F. Wächtler and L. Santos, Ground-state properties and elementary excitations of quantum droplets in dipolar Bose-Einstein condensates, Phys. Rev. A 94, 043618 (2016).
[13] R. N. Bisset and P. B. Blakie, Crystallization of a dilute atomic dipolar condensate, Phys. Rev. A 92, 061603(R) (2015).

[14] R. N. Bisset, R. M. Wilson, D. Baillie, and P. B. Blakie, Groundstate phase diagram of a dipolar condensate with quantum fluctuations, Phys. Rev. A 94, 033619 (2016).

[15] D. Baillie, R. M. Wilson, R. N. Bisset, and P. B. Blakie, Selfbound dipolar droplet: A localized matter wave in free space, Phys. Rev. A 94, 021602(R) (2016).

[16] E. Aybar and M. Ö. Oktel, Temperature-dependent density profiles of dipolar droplets, Phys. Rev. A 99, 013620 (2019).

[17] A. Griffin, Conserving and gapless approximations for an inhomogeneous Bose gas at finite temperatures, Phys. Rev. B 53, 9341 (1996).

[18] A. Boudjemâa, Quantum dilute droplets of dipolar bosons at finite temperature, Ann. Phys. (NY) 381, 68 (2017).

[19] W. Bao, D. Jaksch, and P. A. Markowich, Numerical solution of the Gross-Pitaevskii equation for Bose-Einstein condensation, J. Comput. Phys. 187, 318 (2003).

[20] F. Dalfovo and S. Stringari, Helium nanodroplets and trapped Bose-Einstein condensates as prototypes of finite quantum fluids, J. Chem. Phys. 115, 10078 (2001).

[21] D. S. Jin, M. R. Matthews, J. R. Ensher, C. E. Wieman, and E. A. Cornell, Temperature-Dependent Damping and Frequency Shifts in Collective Excitations of a Dilute BoseEinstein Condensate, Phys. Rev. Lett. 78, 764 (1997).

[22] S. Ronen, D. C. E. Bortolotti, and J. L. Bohn, Bogoliubov modes of a dipolar condensate in a cylindrical trap, Phys. Rev. A 74, 013623 (2006).

[23] I. Ferrier-Barbut, M. Wenzel, F. Böttcher, T. Langen, M. Isoard, S. Stringari, and T. Pfau, Scissors Mode of Dipolar Quantum Droplets of Dysprosium Atoms, Phys. Rev. Lett. 120, 160402 (2018).

[24] F. Böttcher, M. Wenzel, J.-N. Schmidt, M. Guo, T. Langen, I. Ferrier-Barbut, T. Pfau, R. Bombín, J. Sánchez-Baena, J. Boronat, and F. Mazzanti, Dilute dipolar quantum droplets beyond the extended Gross-Pitaevskii equation, Phys. Rev. Res. 1, 033088 (2019). 